\title{
Sample Thickness Limitations in Defocused Electron Probe Ptychography
}

\author{
Arthur M. Blackburn ${ }^{1}$ and Adriaan Frencken ${ }^{2}$
}

1. University of Victoria, Centre for Advanced Materials and Related Technology, Victoria BC, Canada

2. University of Victoria, Department of Chemistry, Victoria BC, Canada

Defocused probe ptychography aims to determine the amplitude and phase shift that a sample object imparts upon an impinging electron beam, using an algorithm that converges modelled and experimentally determined diffraction patterns arising from illuminating the sample in small overlapping regions. One of the most successful and widely used algorithms for this technique is the extended ptychographical iterative engine (ePIE) [1]. This algorithm treats the sample as a simple multiplicative phase object, and so cannot deal with multiple scattering. However, it has been that noted in some situations in electron microscopy, where extinction distances are similar to typical sample thicknesses, some experimental configurations still give useful reconstructions [2]. Within the currently dominant $x-$ ray and optical applications of ptychography, the situation has been treated more comprehensively, and it has been found that propagation within the sample is only adequately considered when the sample thickness $t \leq 5.2 \Delta r^{2} / \lambda=t_{\mathrm{s}}$, where $\lambda$ is the wavelength [3]. The validity of this useful relationship appears to have hitherto not been experimentally investigated in electron microscopy.

In this work we address this, by creating ptychographical reconstructions on a range of samples having thicknesses between $\sim 15 \mathrm{~nm}$ to $100 \mathrm{~nm}$. The experiments were facilitated using recently available highspeed electron detectors synchronized to the beam scan, the prior absence of which has hitherto hindered such explorations. Here, typical $40 \mathrm{~nm}$ square field of view (FOV) regions were illuminated with 1250 overlapping $\sim 5 \mathrm{~nm}$ diameter regions, and diffraction images were collected at 500 frames per seconds or greater, using a single Medipix 3RX chip and Merlin system [4] on a Hitachi HF3300V TEM operated at $300 \mathrm{keV}$. This allowed the above FOV be exposed in 2.5 seconds or less.

Looking first at a test sample of $\sim 5 \mathrm{~nm}$ Au particles on a carbon support, we could easily distinguish the $\mathrm{Au} 022$ plane spacing of $0.142 \mathrm{~nm}$ in the reconstructions (Fig. 1a), giving a resolution that is beyond that of conventional a non-aberration corrected TEM, as expected from earlier studies. In this case, $t / t_{\mathrm{s}} \ll 1$ and the single scattering approximation is valid, leading to high quality reconstructions and clear agreement between ACTEM imaging and electron holography of the same region. Next moving to larger particles, this time $\sim 20 \mathrm{~nm}$ diameter $\mathrm{NaHoF}_{4}$ particles, we were able to resolve $0.18 \mathrm{~nm}$ lattice features in the phase (Fig. 1b) and amplitude reconstructions. Simple application of the relationship above gives $t / t_{\mathrm{s}}=0.6$ and indicates that the reconstruction should be valid. However, in this case there was some disagreement between the HRTEM and the reconstruction, which we attribute to a combination of beam sensitivity of the sample and possibly due to the particle by chance being oriented on-axis. In this case even though the crystal is likely only $\sim 20 \%$ of the electron extinction distance, dynamical (multiple) scattering also possibly becomes significant.

The advantage of orienting crystalline samples off-axis for ptychographic reconstructions is seen when imaging Si transistor devices. Here reconstructing from diffraction patterns collected from an $\sim 70 \mathrm{~nm}$ thickness region beneath a flash memory device, imaged directly down a 100 axis, yielded a poor reconstruction quality, whereas orienting the beam $\sim 2-5 \mathrm{mrad}$ off-axis yielded useful, though not completely artefact free, reconstructions. Low pass filtering of the phase (Fig. 2a) clearly delineates the 
regions of the transistor, and high pass filtering shows the $0.192 \mathrm{~nm} 022 \mathrm{Si}$ planes (Fig. 2b).

However, some repetitive motifs still appear in the reconstruction, which in particular would currently limit the usefulness of the reconstructed lattice, for example as input into geometric phase analysis routines for strain analysis. Part of the reason for the artefacts is the employed ePIE algorithm not accounting for dynamical scattering features appearing in the central disc of the $3-4$ mrad beam half angle convergent beam diffraction patterns, where we note that though the sample thickness is less than $t_{\mathrm{s}}$ given above, the sample thickness is close the Si extinction distance. To accommodate such features in the diffraction patterns likely more advanced multi-slice algorithms will need to be employed [5]. Nonetheless, we conclude that the relationship given by Tsai et al [3] proves useful for electron microscopy, so long as steps are taken to mitigate the effects of dynamical diffraction, such as orienting the sample slightly off-axis if dynamical effects appear or are likely to occur in the sample.

\section{References:}

[1] A M Maiden, J M Rodenburg, Ultramicroscopy 109 (2009), p. 1256

[2] J M Rodenburg, Advances in Imaging and Electron Physics 150 (2008), p. 87

[3] E H R Tsai, et al, Optics Express 24 (2016), p. 29089

[4] R Plackett, et al, Journal of Instrumentation 8 (2013), p. C01038

[5] A M Maiden, P Li, Scientific Reports 8 (2018), p. 2049
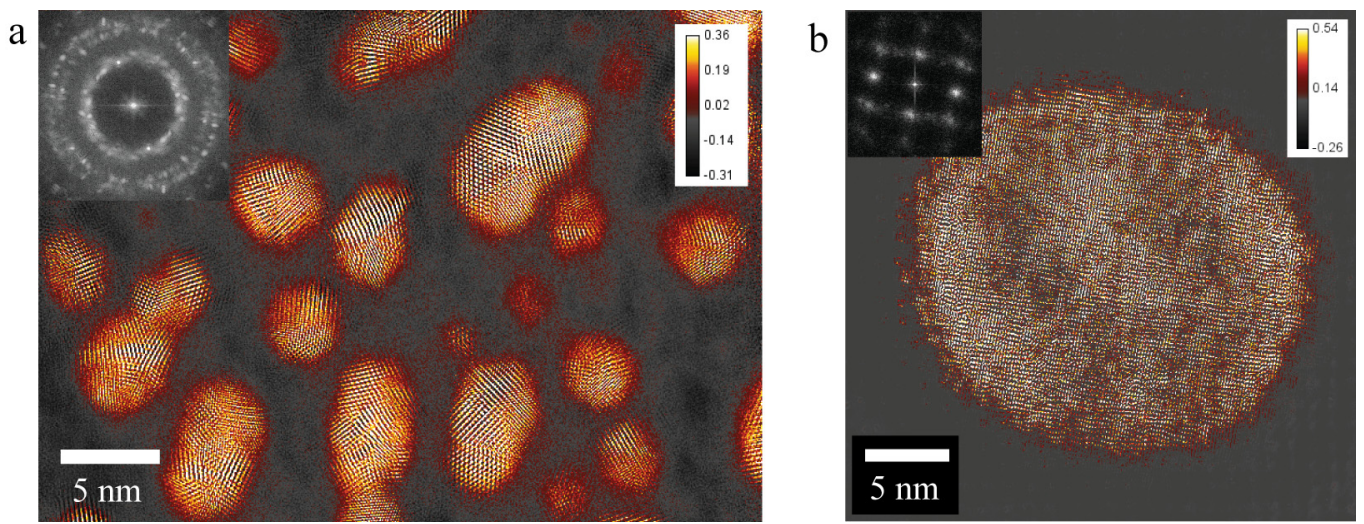

Figure 1. Reconstructed phase images of (a) Au particles and (b) a $\mathrm{NaHoF}_{4}$ particle, both supported on carbon films. Upper right insets show FFT of image. Phase color scale is in radians.

a

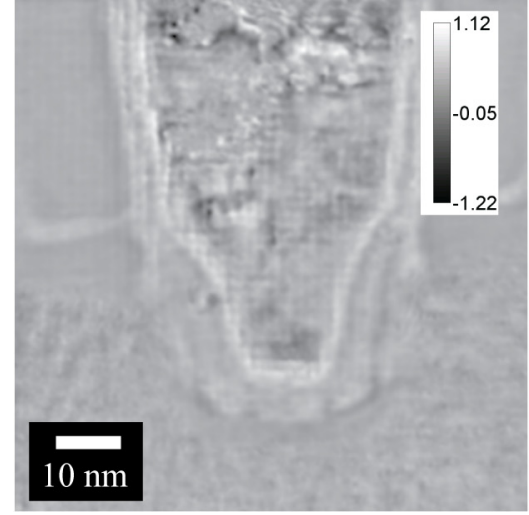

b

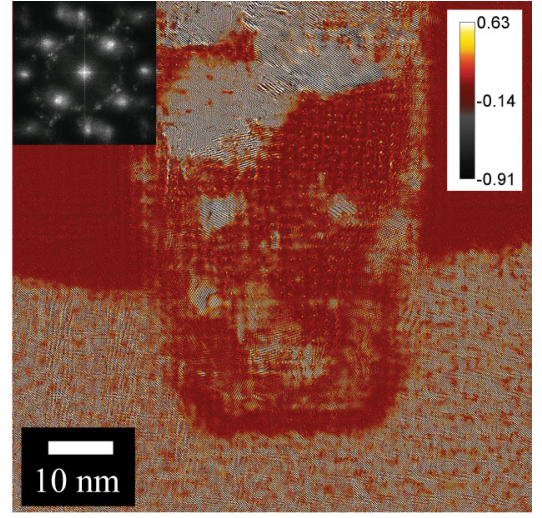

Figure 2. Reconstructed phase of Si transistor region beneath a flash memory stack, separated into its (a) low and (b) high spatial frequency components, inset with an FFT of the image (phase in radians). 